\title{
Una crisis dentro de la crisis: las pésimas relaciones entre los gobiernos republicano y de la Generalitat en 1939
}

\author{
Francesc Vilanova I Vila-Abadal *
}

\begin{abstract}
RESUMEN
ABSTRACT

En 1939, enmedio de la crisis general In 1939, in the middle of the general del final de la guerra civil, estalla una nueva crisis cuyos antecedentes deben encontrarse en los inicios del conflicto, el verano de 1936, y las anomalías constitucionales que se derivaron de aquellos. Los gobiernos de la República (presidido éste por el doctor Juan Negrín) y de la Generalitat (presidido por Lluís Companys) se enzarzaron en una agria polémica acerca de la administración de los fondos económicos del gobierno catalán y su aplicación a la ayuda a los refugiados catalanes. Más allá de estas discusiones, en el fondo de la crisis latian los desencuentros y la desconfianza entre el poder republicano español y un poder autonómico que quería conservar los espacios autonómicos conquistados a lo largo del verano y otoño de 1936. crisis of the final civil war in Spain, a new crisis explodes which has it origins in the early times of the conflict in summer of 1936, and the constitutional anomalies that it generated. The government of the Second Republic (presided by Dr. Juan Negrín) and the government of the catalan Generalitat (presided by Lluis Companys) were confronted about the administration of the catalan government's economic founds and their application to help catalan refugee.
\end{abstract}

Fundació Carles Pi i Sunyer. 


\section{PALABRAS CLAVE}

JARE, Lluis Companys, Juan Negrín, Guerra Civil, Generalitat de Catalunya, Carles Pi i Sunyer, exilio, refugiados, SERE, Segunda República.

\section{KEY WORDS}

JARE, SERE, Lluís Companys, Juan

Negrín, Spanih Civil War, exil,

refugees, Spanish Republic.

En enero de 1942, Carles Pi i Sunyer escribía lo siguiente a Pere Bosch i Gimpera: “[...] resulta una mica estrany, i fins m'arriscaria a dir commovedora, aquesta preocupació de vestal que s'ha despertat en ells ${ }^{1}$ i en molts demòcrates espanyols pel respecte jurídic i la intangibilitat de la Constitució. Sembla que no hagi passat res, o que tots ho haguem oblidat. Vaig votar la Constitució, no li tinc en absolut cap animositat, però cal reconèixer que no va ésser l'instrument que donés estabilitat a la República. I cal reconèixer encara més que no és ara que vulguem prescindirne, que repetidament havia estat ultratjada i vulnerada. No ho fou durant el bienni? No ho fou encara més i fins a un grau irreparable, durant la guerra? No era una demostració de creureho així l'acceptació dels 13 Punts que destruïen la Constitució? Amb la dimissió de l'Azaña, amb la dimissió d'en Martínez Barrio, amb el fraccionament del Govern Negrín, amb la dispersió dels Diputats i la falta de viabilitat dels organismes parlamentaris, no és possible poder fer com a única premissa d'actuació i solucions polítiques un púdic respecte de verge als textos jurídics. I això mateix amb l'Estatut. Que no va ésser rebassat per nosaltres mateixos, al començament de la guerra? Que no va ésser després, al final, desconegut, mistificat i reduit? Però més encara. No hi ha el fet que, en sortir de Catalunya, el govern deixà d'existir com a tal, i tornàrem a concentrar totes les funcions en el President? »?

PI I SUNYER se refiere a los republicanos catalanes "legalistas" (la mayoría de ellos importantes dirigentes d'Esquerra Republicana de Catalunya), que defendian de forma radical la validez del modelo de la II República (con sus textos constitucionales y estatutarios de 1931 y 1932) y su reinstauración en España, frente a las propuestas del Consell Nacional de Londres y, particularmente, de su presidente, Carles PI , SUNYER. El Consell iba en la línea de una III República de carácter federal o confederal, en el contexto de una Europa reorganizada (después de la victoria aliada) según principios federalistas. Sobre estas discusiones y, sobre todo, el papel de PI I SUNYER, puede consultarse: Francesc VILANOVA, "Aproximació al primer exili de Carles PI! SUNYER (19391946)", en Carles Pi i Sunyer (1888-1971), Ajuntament de Barcelona/Fundació Carles Pi i Sunyer, 1995, pp. 191-233; Daniel Diaz Escultes, El catalanisme politic a l'exili (1939-1959), Ed. La Magrana, Barcelona, 1991; Carles PI I SunYer, Reports (1939-1943), Fundació Carles Pi i Sunyer, Barcelona, 1993; y Conferències al Casal Català de Londres (1941-1943), Fundació Carles Pi Sunyer, Barcelona, 1994.

Carta de Carles PI I Sunyer a Pere Bosch i Gimpera, 30 enero 1942. Para contextualizar esta carta, véase Francesc VILANOVA, "Nota a l'edició, en Carles Pi i Sunyer, Catàlunya en la guerra civil espanyola, Fundació Carles Pi i Sunyer, Barcelona, 1993, pp. 9-24. 
Una crisis dentro de la crisis: las pésimas relaciones entre los gobiernos...

Aunque se refería a una situación específica del exilio republicano de 1939, con este texto Pi situaba algunos de los puntos cruciales de la profunda crisis que dividió el exilio español y, sobre todo, marcó las distancias políticas casi insalvables entre las instituciones republicanas y catalanas. Pero si Pi ponía sobre el papel algunos de los elementos que, desde 1936, habían cambiado radicalmente los equilibrios y el entramado institucional y constitucional de la II República, hay otra visualización de la ruptura, de las mutuas desconfianzas nacidas y alimentadas con la guerra, que se plasmaron en el conocido episodio de la salida por separado de las autoridades republicanas, el 5 de febrero de 1939, del territorio catalán en dirección a Francia ${ }^{3}$. Manuel Azaña, acompañado por Juan Negrín, salió en dirección a la frontera a las cinco de la madrugada. A las ocho, hora convenida por Lluís Companys y el mismo Azaña, salieron los dirigentes catalanes y los vascos (encabezados por el lehendakari José Antonio de Aguirre) que, sorprendidos, descubrieron que Azaña ya había partido ${ }^{4}$. Es la mejor imagen de la profunda división existente entre las diferentes instituciones y representaciones políticas de la II República.

Ciertamente, los problemas entre el gobierno central y el catalán venían de lejos, desde casi los inicios de la guerra civil. Después de los hechos de mayo de 1937 en Barcelona, el gobierno central asumió nuevamente las

3 Véase, por ejemplo, Josep BENET, Exili i mort del president Companys, Ed. Empúries, Barcelona, 1991, pp. 39-43; Julián Zugazagoltia, Guerra y vicisitudes de los españoles, Ed. Crítica, Barcelona, 197737, pp. 526-527; Diego Martinez. BarRio, Memorias, Ed. Planeta, Barcelona, 1983. pp. 404-405; Manuel AZAÑA, Memorias politicas y de guerra, vol. II, Ed. Crítica, Barcelona, 19814, pp. $445-448$.

4 Además del durísimo comentario que Negrin dedicó a Azaña, según la versión recogida por Julián Zugazagoitia (Guerra y vicisitudes..., p. 527), el presidente del Consejo de Ministros también dedicó unas palabras al grupo vasco-catalán que salió unas horas más tarde: "Lo que no podia esperarme es que a mi ingreso fuese a tropezar con Aguirre y Companys. Los más sorprendidos han sido, naturalmente, ellos, que han debido sospechar que yo abandonaba el territorio nacional, sin notificarles mi decisión. El juego de palabras ha sido precioso. Se han ofrecido a regresar conmigo, pero me he negado. Ausentes de Cataluña, tengo una preocupación menos" (Julián Zugazagoitia, Guerra y vicisitudes... p. 527). Frente a esta versión contamos con el testimonio directo de uno de los integrantes del grupo vasco-catalán, Carles P। I SUNYER: "Quan feia ja un temps que caminàvem veiérem venir pel sender Negrín. Tot sol. De moment ens estranyà trobarlo; però ell ens digué que venia d’acompanyar a Azaña i la seva esposa. El meu nebot Cèsar que, junt amb Cristià Cortés, sortiren de nit del mas per a passar la ratlla a la matinada, ens contà més tard com, just arribar a les llles, veieren venir Azaña, la seva esposa i Negrín que els acompanyava. Negrín duia del braç doña Lola; el rostre d'Azaña era seriós, amb solcs de preocupació. En el moment d'acomiadar-se, Negrín besà la mà de la senyora i li digué: "Hasta pronto, en Madrid". Un grup de refugiats cridà: "¡Viva la República!"; Azaña i la seva esposa pujaren al cotxe que els esperava i Negrín se'n tornà muntanya amunt. Venia d'acomplir aquest gest cavallerós quan el trobàrem. Amb nosaltres estigué, si no arribant a cordial, sí amistós. Ens acomiadàrem expressant els mutuals desitjos de bona sort en les hores incertes que teníem al davant" (Carles PI SUNYER, La guerra, 1936-1939. Memòries, Ed. Pòrtic, Barcelona, 1987, p. 243). 
competencias de orden público y, a partir de este momento, se estableció una dura pugna entre la Generalitat de Catalunya y el gobierno de la República sobre los ámbitos competenciales de cada una de los dos instancias. Desde las industrias de guerra hasta la enseñanza, pasando por el patrimonio artístico. El balance final fue un gran poso de amargura, una quiebra en la confianza entre las diferentes instituciones y una larga lista de agravios, que se pusieron encima de la mesa inmediatamente después de cruzar la frontera ${ }^{5}$.

El primer problema de la Generalitat, en aquellos días de febrero de 1939, era que sus dirigentes y los restos de sus instituciones salían de Cataluña sin recursos económicos. Entre diciembre de 1938 y marzo de 1939 , el Departamento de Hacienda de la Generalitat fue entregando diferentes partidas económicas (en divisas, moneda española, objetos de joyería, valores del Tesoro, etc.) al gobierno de la República, en base a las resoluciones del mismo gobierno republicano y en el bien entendido que estos fondos servirian para sufragar las necesidades de los refugiados catalanes en Francia, tanto para su establecimiento en este país como para financiar su traslado a América u otros países, y también para el mantenimiento de las actividades culturales catalanas en el destierro ${ }^{6}$. Teóricamente, para

\footnotetext{
5 Para no ofrecer una prolija bibliografía sobre estas cuestiones, remito al lector a los trabajos, ya citados, de Carles P। I SUNYER (Catalunya en la guerra civil espanyola, La guerra, 19361939. Memòries) y Julián Zugazagoitia (Guerra y vicisitudes de los españoles). También es muy útil, para el estudio de los conflictos competenciales en el ámbito cultural, el trabajo de María Campillo, "Carles Pi i Sunyer, Conseller de Cultura en temps de guerra", en Carles Pi i Sunyer (1888-1971), ya citado, pp. 155-190.

- Carles PI I Sunyer, La guerra, 1936-1939. Memòries, pp. 224-2254. Con respecto a la entrega de los fondos económicos de la Generalitat al gobierno de la República (bien al Banco de España, bien al Ministerio de Economía y Hacienda), un primer balance sería el siguiente (elaborado a partir de las copias mecanografiadas de los documentos originales de la Conselleria d'Hisenda de la Generalitat, conservadas en el Arxiu Carles Pi i Sunyer):

- Hasta el 9 de noviembre de 1938: entregados 34.594'5 títulos de valores de cotización internacional, en posesión de la Generalitat, al Banco de España.

- Período 10-31 diciembre 1938: entrega de 270 lingotes de plata al Banco de España.

- 24 enero 1939: entrega de 699 lingotes de plata (11.890 kiłos) al Banco de España.

- Período 23 enero-2 febrero 1939: entrega de 73 cajas al Ministerio de Economia y Hacienda (en Figueres), con “joies, perles, metalls preciosos i divises rebuts per la Generalitat de Catalunya, procedents d'organismes públics, banca, particulars, partits polítics i sindicats".

- 29 enero 1939: entrega de divisas en posesión de la Generalitat al Tesoro de la República (liras del Vaticano, Reichmarks, libras esterlinas, dólares, etc.).

- 30 enero 1939: entrega de 59 sacos conteniendo «moneda española de plata, metal dorado, cupro-níquel y cobre, procedente de la existencia en Caja del servicio de la Caixa de Dipòsits Consignacions de la Generalitat de Catalunya», por un valor total de 229.699'50 ptas.

- 30 enero 1939: “En esta fecha, la Tesorería General de la Generalitat de Catalunya entrega al Tesoro Público, en cumplimiento de disposiciones del Gobierno de la República, ciento tres kilogramas, setecientos siete gramos (103'707 K.gs.) peso bruto, de plata en lingotes de diversos
} 
reforzar esta situación, se estableció una nueva instancia política, el llamado Consejo de los Cinco Presidentes. Éste tenía que ser un organismo integrado por los presidentes de la República, del Consejo de Ministros, de las Cortes, de la Generalitat y del gobierno vasco ${ }^{7}$. Según el testimonio de $\mathrm{Pi}$ i Sunyer, tanto Azaña como Negrín aceptaron inicialmente la creación de este organismo, después de eliminar algunos recelos y desconfianzas.

Evidentemente, el Consejo de los Cinco Presidentes y la entrega de los fondos económicos de la Generalitat estaban vinculados y, además, representaban un salto cualitativo de una gran magnitud. Los dirigentes catalanes confiaban plenamente en esta nueva instancia, a pesar de que no existía ninguna formalización legal sobre la que apoyar futuras reivindicaciones. Con la perspectiva del tiempo, parece evidente que aquello no podía funcionar de ninguna manera; es más, posiblemente ni los mismos protagonistas estaban absolutamente convencidos de que este Consejo pudiera ser operativo. Entonces, ¿por qué se llegó a plantear y, todavía más, los dirigentes catalanes confiaron en que este Consejo garantizaría la supervivencia económica del exilio catalán? En el contexto de desintegración del mundo republicano, con los enfrentamientos públicos y abiertos entre Manuel Azaña y Juan Negrín, la situación del gobierno catalán era extraordinariamente débil. Desde el verano de 1937, las tensiones con el gobierno de la República habían derivado en una progresiva intervención del gobierno central en los asuntos catalanes. Sin territorio propio, sin fuerzas

pesos y tamaños y en monedas francesas, recibidos por la Generalitat de Catalunya de Particulares"

- 2 febrero 1939: entrega de cuatro cajas y un saco, conteniendo 443.053'805 pesetas-oro.

- 2 febrero 1939: 449 monedas de oro, de valor numismático.

- 2 tebrero 1939: "En esta fecha, la Tesorería General de la Generalitat de Catalunya entrega al Tesoro Público, en cumplimiento de disposiciones del Gobierno de la República, aproximadamente Kilogramos 83'871.812 [...] de oro fino, recibidos por la Generalitat de Catalunya, de Organismos Públicos, Partidos Políticos, Sindicales y Particulares".

- 2 febrero 1939: “...una olla de cobre conteniendo Bisutería diversa, con un peso bruto de Kilogramas veintiocho, gramos ochocientos $\left(28^{\prime} 800 \mathrm{Kgs}\right.$.), recibida por la Generalitat de Catalunya, de Organismos Públicos, Partidos Políticos, Sindicales y Particulares".

- 22 febrero 1939: ingreso en la gerencia del Banco de España, en París, de 10.692.763 ptas., remanente de diferentes operaciones de la Tesoreria General de la Generalitat, la Comisión de Industrias de Guerra y el Servicio de Divisas y Valores y Metales Preciosos.

Una información complementaria sobre la entrega de fondos de la Generalitat al gobierno de la República, basada en recuerdos de terceras personas, en Joan Sauret, L'exili politic català, Ed. Aymà, Barcelona, 1979, p. 15. Véase, también, Daniel DiAz EsculiEs, Entre filferrades. Un aspecto de l'emigració republicana des Paisos catalans (1939-1945), La Magrana, Barcelona, 1993, pág. 21 y págs. 148-158.

Es decir: Manuel azaña, Juan Negrin, Diego Martinez Barrio, Lluís Companys y José Antonio De Aguirre. Véase Carles PI I Sunyer, La guerra, 1936-1939. Memóries, pp. 225-226; Daniel DiAZ Escul IES, El catalanisme polític a l'exili (1939-1959), pp. 45-46. 
armadas, con su población desmoralizada, sin apoyos internacionales que pudieran ser utilizados como una garantía de actividades futuras, la única salida era esperar algo de "lealtad constitucional e institucional" entre los dos gobiernos para organizar el inminente exilio. Lluís Companys, presidente de la Generalitat, se mostró muy firme sobre el significado de esta "lealtad constitucional e institucional»; a pesar de ser consciente de su debilidad política (en lo individual y en lo colectivo), Companys afirmaba que «el entonces Presidente del Gobierno, Sr. Negrín, me prometió, en el mes de Enero, dar cauce a tales obligaciones [la necesidad de proveer a los deberes contraídos con quienes lucharon lealmente a nuestro lado por la República] mediante un organismo en el que se integrarían las representaciones institucionales de la República y de los paises autónomos comprendidos en nuestro régimen constitucional, de modo que pudieran mantenerse unos y otros, sin decaer en su personalidad y con la garantía de compartir la administración de los intereses de todos los emigrados políti$\cos ^{8}{ }^{8}$. No quedaba claro si Companys se refería a un servicio de ayuda a los refugiados o bien al Consejo de los Cinco Presidentes, pero al menos puede deducirse que, cuando se planteó la creación del Consejo (en los primeros dias de febrero), los dirigentes catalanes tenían muy presente la promesa precedente de Negrín en Barcelona.

Partiendo de esta base, el gobierno catalán entregó sus fondos económicos al gobierno central y a cambio recibió una subvención, "como prenda de una futura ayuda", de 150.000 francos para el auxilio inmediato a los refugiados que estaban cruzando la frontera ${ }^{9}$. Companys continuaba haciendo balance de la situación: "Sin otra subvención, invertimos en estas atenciones la totalidad de los menguados saldos de la Generalidad en el extranjero, siempre en espera de las aportaciones prometidas y bajo la presión de necesidades angustiosas de los refugiados,

\footnotetext{
- Carta del presidente de la Generalitat [Lluis COMPANYS] al presidente de la JARE [Lluis Nicolau d'Olwer], París, 17 agosto 1939. En 1945, los dirigentes catalanes volvieron a insistir en Este punto de la constitución del Consejo de los Cinco Presidentes: "...antes de abandonar el territorio catalán, en las reuniones celebradas en los castillos de Perelada y de Figueras, y en las que intervinieron personalidades republicanas, demasiadas de ellas (AZAÑA, COMPANYS, ZUGAZAGOITIA) trágicamente arrebatadas a la vida, se convino en estructurar la alta dirección de la obra republicana en el destierro a base del llamado Consejo de los cinco Presidentes (de la República, de las Cortes, del Gobierno, de Cataluña y de Euzkadi), el cual deberia llevar la alta dirección de la obra republicana tanto en cuanto afectase su mantenimiento en el destierro, como a la reconquista de la República" (Informe del presidente de la Generalitat [Josep IRLA] al presidente del Consejo de Ministros de la República española [José GIRAL], diciembre 1945).

Carta del presidente de la Generalitat [Lluís COMPANYS] al presidente de la JARE [Lluís NICOLAU D'OLWER], París, 17 agosto, 1939 Esta partida económica, entregada por el gobierno republicano, fue transferida el 2 de febrero de 1939.
} 
abandonados en los campos por la administración española. En el mes de marzo, la Generalidad había agotado sus recursos y cerraba, con fecha 11 de dicho mes, sus cuentas. Multiplicados después los requerimientos al Sr. Negrín y siempre sin respuesta o correspondidos con promesas incumplidas, la modesta organización de ayuda a los catalanes tuvo que apelar a los centros de América y a la hospitalaria amistad de los intelectuales franceses, que han contribuido con esfuerzos y aportaciones particulares al sostenimiento de refugios en los cuales tienen provisional acomodo unos centenares de hombres representativos de las instituciones culturales, políticas y administrativas de Cataluña. Al sostenimiento de esta obra se han dedicado también principalmente los fondos recibidos por Esquerra Republicana de Catalunya, del Comité de Ayuda a España, y siendo en mucho insuficientes, ante el dilema de mantener a todo trance los servicios organizados o de lanzar a los campos de concentración a los diputados del Parlamento catalán -para los que no se ha podido aun obtener el subsidio que perciben comandantes, jueces y alcaldes de ciudades provinciales-, a los miembros del Ayuntamiento de Barcelona y otras capitales, a los académicos, miembros del Institut d'Estudis Catalans, profesores, artistas, escritores -para los cuales tampoco se ha podido obtener ningún subsidio-, me he visto obligado a recurrir al crédito de entidades y personas solidarizando mi propia garantía con la que nuestros colaboradores franceses y catalanes han podido arbitrar" ${ }^{10}$. Antes de terminar, Companys todavía tenía tiempo de criticar a los vascos: "Contrasta esta falta de los más imprescindibles medios para atender las necesidades de los catalanes, con la pluralidad de órganos y publicaciones sectarias que aparecen al servicio de banderías y grupos, con recursos de origen desconocido para nosotros. [...] Y contrasta asimismo nuestra honrada pobreza con la relativa abundancia en que justamente vive la administración vasca, atendida con una importante subvención mensual cifrada en varios millones, que recibe de la administración regida por el Sr. Negrín desde el mes de abril, no obstante ser la administración vasca de volumen muy inferior a la catalana» ${ }^{11}$.

10 Carta del presidente de la Generalitat [Lluis COMPANYS] al presidente de la JARE [Lluís N/COLAU D'OLwEr], París, 17 agosto 1939. A raíz de las reuniones del Comité de Ayuda España, de finales de marzo de 1939. PI I SUNYER, ante la dificultad para conseguir subvenciones, apuntó: "No és possible obtenir un prés-tec dels bascos per a despeses inicials de 500.000 francs, a retornar amb les aportacions del Govern de la República o de les subvencions i amb la garantia moral d'unes personalitats catalanes?" (Comité de Ayuda a España. Notes de Carles Pi i Sunyer [30 març-1 abril 1939j).

Carta del presidente de la Generalitat [Lluís COMPANYS] al presidente de la JARE [LIuís NICOLAU D'OLWER], París,17 agosto 1939. 
En medio de todo ello, el 5 de abril de 1939 Companys tuvo una entrevista con Juan Negrín, en la que actuó como testigo Diego Martínez Barrio. Esta reunión tenía lugar a los pocos días de la polémica constitución del SERE (Servicio de Emigración de Republicanos Españoles) en su configuración definitiva ${ }^{12}$, y después de la primera gran reunión de la Diputación Permanente de las Cortes Republicanas que tenía encima de la mesa dos asuntos fundamentales: la dimisión de Manuel Azaña y su substitución al frente de la presidencia de la República y la discusión alrededor de la legitimidad del gobierno Negrín ${ }^{13}$. Pero también en estos días se había producido el famoso incidente del Vita, que daría nacimiento a la Junta de Auxilio a los Refugiados Españoles (JARE) ${ }^{14}$. La confusión era absoluta. El enfrentamiento político en el mundo republicano estaba llegando al paroxismo.

El golpe definitivo se dio a finales de julio, en la reunión de la Diputación Permanente de las Cortes republicanas, en la que se desautorizó finalmente al gobierno Negrín ${ }^{15}$. A partir de aquí, y con la disponibilidad del «tesoro" del Vita y la actitud de Indalecio Prieto, se puso en marcha la JARE (Junta de Auxilio a los Refugiados Españoles) ${ }^{16}$. Para los catalanes, el nuevo organismo era una posibilidad única para conseguir lo que Negrín les habia estado negando desde el mes de enero: una ayuda fluida y suficiente para mantener una mínima infraestructura cultural y de auxilio para los refugiados catalanes. Pero el camino para llegar hasta aquí no fue tan sencillo.

Ante los resultados de la reunión de la Diputación de Permanente, la minoría catalana (es decir, el grupo de Esquerra Republicana de Catalunya) empezó por denunciar la profunda división en el campo republicano, algo evidente y de todos sabido, sobre todo después de conocerse la dimisión de Manuel Azaña y la crisis institucional consiguiente, el final de la guerra en Madrid (con el golpe del coronel Casado), las primeras cartas cruzadas entre Indalecio Prieto y Juan Negrín, etc. A partir de aquí, los republicanos catalanes jugaron la carta pragmática: estarían presentes en los dos organismos de ayuda (SERE y JARE) para asegurarse la ayuda para los refugiados catalanes; però, añadían, "si aquest règim complicat i

\footnotetext{
12 Véase, por ejemplo, Javier RuBıo, La emigración de la guerra civil de 1936-1939, Ed. San Martín, Madrid, 1977, vol. I, pp. 130-139.

13 José M. DeL VALLE, Las instituciones de la República en el exilio, Ed. Ruedo Ibérico, Paris, 1976, pp. 18-31.

${ }_{14}$ José M. Del Valle, Las instituciones de la República en el exilio, pp. 32-44. También Javier Rubio, La emigración de la guerra civil..., vol. I, pp. 139 y ss

15 Javier RuBı, La emigración de la guerra civil..., vol. II, pp. 494-495

16 José M. DEL VALLE, Las instituciones de la República en el exilio, p. 41.
} 
artificiós es fa impossible, si la violència de l'antagonisme ho impedeix, i se'ls posa en el dilema de declararse bel.ligerants en una lluita que no senten, aleshores es reserven la facultat de prendre les resolucions que creguin escaients, afirmant la persistència intangible dels drets dels republicans catalans que no poden ésser afectats per les repercussions de les lluites entre grups polítics...» 17.

La posición catalana (estar en los dos sitios a la vez, un poco a la expectativa de lo que pudiese suceder) signficaba jugar algunas cartas que facilitasen esta posición. La primera, y más evidente, era la de tener personas de confianza en el nuevo organismo de ayuda. La presidencia de Lluís Nicolau d'Olwer ya era un buen augurio. Además, se intentó la incorporación como secretario de Eduard Ragasol (uno de los principales dirigentes de Acció Catalana, bien conectado con ambientes políticos franceses). Tras el fracaso de esta canditatura, ERC defendió la propuesta de Jaume Miravitlles (periodista, ex-responsable del Comissariat de Propaganda de la Generalitat) y Josep Quero Molares (subsecretario en diferentes gobiernos republicanos) ${ }^{18}$. A pesar de que ninguna de las propuestas salió adelante (finalmente, el secretario de la JARE sería Carlos Esplá), sí que se constató una mejoría substancial en las relaciones entre los catalanes y las autoridades republicanas con capacidad de decisión y actuación. A finales de agosto, la presidencia de la Generalitat recibió ya unas primeras cantidades para hacer frente a las necesidades de los refugiados bajo su custodia.

De las grandes cuestiones político-constitucionales de los años 19361937 a regatear un acuerdo satisfactorio para ayudar a los refugiados en 1939 , las relaciones entre los gobiernos catalán y el republicano se habian desarrollado en un difícil equilibrio, envenenado por la guerra, las confusiones y la catástrofe del hundimiento de 1939. Ciertamente, los debates de 1939 se produjeron alrededor de cuestiones que poco tenían que ver con una discusión profunda sobre modelos políticos de futuro o las relaciones entre Cataluña y el resto de España. En 1939, la dramática situación de los republicanos no estaba para debates de esta enjundia. Pero ello no debe hacernos olvidar que, detrás de la insistencia de la presidencia de la Generalitat para preservar la máxima autonomía en la ayuda a "sus" refugiados, había una posición política claramente definida: recuperar los niveles de gestión autonómica, si no de 1936, sí los establecidos

\footnotetext{
Nota de la minoría catalana, reproducida por Carles PI। SUNYER en Memòries de l'exili. l: El Consell Nacional de Catalunya (1940-1945), Curial, Barcelona, 1978, p. 23.

18 Carta de Josep Tarradellas a Carles PI I Sunyer, Paris, 21 agosto 1939
} 
en 1932 que la Generalitat consideraba que el gobierno Negrín había vulnerado desde otoño de 1937. Los «males» de la guerra se proyectaron a lo largo de 1939 en todos los niveles políticos e institucionales y si algunas heridas se cerraron, no todas, fue porque el inicio de la segunda guerra mundial cambió radicalmente la situación y el contexto. 